\title{
Evolución de factores competitivos de la empresa familiar ante cambios en la propiedad: el caso mexicano estudiado en Estado de México, Tamaulipas, Quintana Roo y Puebla
}

\section{Evolution of competitive factors of the family business due to ownership changes: an aggregate study including the states of México, Tamaulipas, Quintana Roo and Puebla.}

\author{
Argentina Soto Maciel ${ }^{\mathrm{a}} \cdot$ María Isabel de la Garza Ramos $^{\mathrm{b}} \cdot$ José Luis Esparza Aguilar $^{\text {c* }} \cdot$ Juan Manuel \\ San Martín Reyna ${ }^{\mathrm{d}}$ \\ ${ }^{a}$ Universidad Anáhuac, (México), ${ }^{b}$ Universidad Autónoma de Tamaulipas (México), ${ }^{c}$ Universidad de Quintana \\ Roo (México), ${ }^{d}$ Universidad de las Américas, Puebla (México)
}

D A T O S A R T ÍC U L O

Historial:

Recibido 18-07-2014

Aceptado 26-04-2015

Palabras clave:

Empresa Familiar

Teoría de los recursos y las

capacidades

Factores competitivos

Cambio de propiedad

Códigos JEL:

L1, L2, M1, M2

A R T I C LE IN F O

Article history:

Received 18-07-2014

Accepted 26-04-2015

Keywords:

Family business

Resource-Based View

Competitive factors

Change of ownership.

JEL codes:

L1, L2, M1, M2

\section{RES UMEN}

El interés creciente por hacer perdurar las actividades económicas de la empresa familiar conduce gradualmente a realizar mayores esfuerzos de investigación con la finalidad de mejorar su comprensión y conocimiento para así reducir su vulnerabilidad. El presente trabajo se inscribe en la Teoría de los Recursos y Capacidades y se interesa en conocer la evolución de algunos factores internos a la empresa familiar relacionados con la competitividad y el cambio de propiedad en cuatro estados de México. Este documento es el resultado del trabajo colectivo de una red de investigación, cuyos autores pertenecen a la Universidad Anáhuac, Universidad Autónoma de Tamaulipas, Universidad de Quintana Roo y Universidad de las Américas.

\section{A B S T R A C T}

The growing interest to guarantee the economic success of family businesses, gradually leads to bigger efforts on research, seeking for a better understanding to reduce their vulnerability. The Theory of Resource-Based View is the framework in which this paper is based on, and its main objective is to know the evolution of some internal factors, within the family businesses, that are related to competitiveness and ownership changes, in four Mexican states. This document is the result of the collective work of a network of research, whose authors belong to the University Anáhuac, University of Tamaulipas, University of Quintana Roo and University of the Americas.

\footnotetext{
* Autor de contacto.
}

Correos_electrónicos: argentina.soto@anahuac.mx,_Idelagarza4@gmail.com,_jlesparza@uqroo.edu.mx* , juanm.sanmartin@udlap.mx 


\section{Introducción}

Hasta los años 80's, la tendencia estratégica dominante de las empresas privilegiaba su adaptación a las características estructurales de su contexto competitivo, que era relativamente estable. Progresivamente, la inestabilidad de los mercados se consolidó, el fenómeno de la globalización conllevó la apertura de los mercados y el incremento de la competencia, lo que aunado al acelerado desarrollo tecnológico, planteó un escenario competitivo contrastante al previo. En este nuevo contexto, resurge el interés por las estrategias fundadas en la gestión de los recursos de las empresas. En 1959, Penrose afirmaba que "la empresa tiene un carácter único a partir de la heterogeneidad de los servicios productivos disponibles o potencialmente disponibles". Contrariamente a la concepción neoclásica a través de la cual se considera que los recursos son accesibles en el mercado, la Teoría de los Recursos y las Capacidades reconoce que se trata de factores específicos, poco transmisibles y valiosos en función al tiempo necesario para desarrollarlos. A partir de esta concepción, la construcción de ventajas competitivas en mercados imperfectos tiene su fundamento en la gestión eficaz de esos factores internos y distintivos. En su inicio, el estudio de los recursos estratégicos para la empresa enriqueció la creatividad semántica del término, Métais (2004) lo muestra al censar una veintena de definiciones diferentes. En ese proceso, los recursos han sido considerados como activos invisibles (Itami, 1987), capacidades organizacionales (Stalk, Evans, Shulman, 1992), competencias centrales (Hamel, Prahalad, 1990), red de recursos (Black, Boal, 1994) o estructuras organizacionales (Williamson, 1996). Sin embargo para dos de sus precursores, los recursos "son activos tangibles e intangibles poseídos o controlados por la empresas" (Wernerfelt, 1984)

Según Barney (1991) "todos los activos, capacidades, procedimientos, informaciones, conocimientos, etc. controlados por la empresa y que le permiten inventar y diseñar estrategias eficaces y eficientes".

Bajo esta perspectiva se percibe a la empresa como un conjunto único de recursos y capacidades idiosincráticas, donde la primera tarea de la administración es maximizar el valor de la empresa a través del despliegue óptimo de recursos y capacidades existentes, así como proyectando en el futuro el desarrollo de sus propios recursos (Grant, 1996). Dentro de los fundamentos teóricos de esta perspectiva se encuentran diferentes componentes; recursos, competencias, capacidades organizacionales y activos estratégicos (Teece, Pisano, Shuen, 1997; Amit, Schoemaker, 1993). Mientras que los recursos estratégicos internos o externos (tangibles o intangibles) son activos específicos de la empresa (Grant, 1991), las competencias que son internas se relacionan con la capacidad de la empresa para combinar dichos recursos (Grant, 1991) y las capacidades organizacionales con las rutinas dinámicas a efecto positivo sobre la eficiencia y flexibilidad de la organización (Winter, 1987). Amit y Schoemaker (1993) reconocen que los activos estratégicos son el conjunto de recursos y capacidades difíciles de intercambiar, transferir, imitar, raros y que constituyen la ventaja organizacional de una empresa. Estos activos pueden estar compuestos entre otros elementos de capacidades tecnológicas, acceso a diferentes canales de información, relaciones con distribuidores o proveedores, capacidad de realizar investigación y desarrollo o inclusive tener una cierta reputación. Para Arrègle (1995) se trata de una combinación compleja de habilidades específicas a la empresa que le permiten satisfacer mejor y de manera durable cierto nicho del mercado.

Así la ventaja competitiva es el resultado de una combinación de recursos movilizando procesos organizacionales y saber-hacer colectivo (Amit y Schoemaker (1993). Los factores considerados para la elaboración de este trabajo debido a su valor estratégico se relacionan con los recursos humanos, la calidad, la innovación y la tecnología.

A pesar de que a nivel mundial son múltiples los estudios que sobre la empresa familiar se han hecho diferenciándola de la empresa no familiar (Claver, Rienda, y Quer, 2006; Allouche, Amann, Jaussaud y Kurashina, 2008; Barnett, Eddleston y Kellermanns, 2009), la noción aún carece de unanimidad (Ward 1988; Wortman 1994; Gallo, 1995; Cabrera y García, 1998; Neubauer y Lank, 1999). Para los fines de este estudio se le considerará empresa familiar a aquella cuya propiedad y control se encuentran, en su mayoría, en manos de una familia y existe el deseo expreso de continuidad de la misma.

Con estos argumentos previos, se ha planteado la siguiente pregunta de investigación: ¿existen factores internos relacionados con la competitividad de las empresas familiares mexicanas considerando diferentes perspectivas en su cambio de propiedad?

Soto Maciel, A., de la Garza Ramos, M.I., Esparza Aguilar J.L. y San Martín Reyna, J.M. (2015). Evolución de factores competitivos de la empresa familiar ante cambios en la propiedad: el caso mexicano estudiado en Estado de México, Tamaulipas, Quintana Roo y Puebla. Revista de Empresa Familiar, 5 (1), 53-64. 


\section{Objetivos}

En la investigación nos interesamos en conocer y comparar la situación en la que se encuentran algunos factores internos relacionados con la competitividad de las empresas tomando en consideración diferentes perspectivas en el cambio de propiedad de la empresa familiar en México.

\section{Marco Teórico}

A continuación abordaremos los temas centrales sobre los cuales gira la investigación.

Recursos humanos: Algunos autores han asociado positivamente el tema de recursos humanos con la productividad (Huck y McEwen, 1991; Warren y Hutchinson, 2000). El estudio se interesa en conocer el estado observado en temas como la organización del personal, la satisfacción, la rotación del personal o el ausentismo laboral.

Calidad: La calidad es entendida como la adecuación de las características del producto o servicio a las necesidades del cliente. La calidad ha estado frecuentemente asociada a la competitividad y al éxito de las empresas (Viedma, 1990; Luck, 1996; Camelo 1999). El estudio se interesa en conocer la evolución de la calidad del producto o del servicio, de los procesos operativos internos, la satisfacción de los clientes, la rapidez de adaptación a las necesidades del mercado, la imagen de la empresa y de sus productos o servicios, la participación en el mercado, la rentabilidad y la productividad.

Innovación: La innovación se considera la mejora o los cambios frecuentemente incrementales en distintas áreas (producto, proceso, distribución) que han sido realizados por las empresas en los últimos dos años (North et al., 2001). Para las empresas, la innovación y la tecnología son fuente de ventajas competitivas sostenibles en el tiempo (Vermeulen, 2004) y determinantes en su crecimiento económico (Alvarez y García, 1996; Camelo, 1999; Van Auken, 2008). El estudio identifica por ejemplo, mejoras que las empresas han tenido en productos o servicios existentes, en la comercialización de nuevos productos o servicios, en la gestión empresarial, en los procesos de producción, en las tecnologías de información y comunicación, en la gestión de la calidad, en las compras y en las ventas.

Tecnología: La tecnología es entendida como el conjunto de conocimientos, formas, métodos, instrumentos $y$ procedimientos que permiten combinar los diferentes recursos y capacidades en los procesos productivos y organizativos para lograr que estos sean eficientes (Donovan, 1996). La incorporación de nuevos sistemas y tecnologías de la información facilitan la planificación, fomentan el desarrollo tanto de programas de producción, mejora el control de los procesos (Huerta et al., 2003). Así se relaciona positivamente el uso de nuevas tecnologías de la información y comunicación para la gestión de la empresa con su nivel competitivo (Viedma, 1992). El estudio se interesó en conocer la posibilidad de contar con conexión a Internet, correo electrónico, pagina WEB, así como la utilización de tecnología para realizar mercadotecnia, mantener relaciones con proveedores y clientes, realizar compras y ventas por Internet, o el uso de las redes sociales dentro de su gestión.

\section{Método}

El diseño general de la muestra tiene como base los principios del muestreo no probabilístico por cuotas, ya que se han seleccionado a las empresas siguiendo determinados criterios de propiedad familiar y procurando, en la medida de lo posible, que la muestra sea representativa. Debido a que el estudio se refiere a empresas que están cerca de la sucesión o que, al menos la están contemplando en sus planes, el diseño no puede hacerse de otra forma sino a través de una selección directa. En el estudio participaron un total de ochenta y cinco empresas familiares distribuidas equitativamente en los Estados de México, Tamaulipas, Quintana Roo y Puebla.

La metodología utilizada para la recogida de información ha sido la encuesta (Hernández, Fernández y Baptista, 2006). El cuestionario se diseñó tomando en cuenta los trabajos de Lansberg y Astrachan (1994), así como la revisión teórica y empírica de diversos estudios relacionados con el objeto de estudio. El cuestionario definitivo consta de 17 preguntas organizadas en varios bloques temáticos. En este trabajo se explotan dos bloques: uno enfocado a la evolución de algunos aspectos de eficiencia organizacional; el otro, orientado al progreso experimentado por la empresa en relación a la innovación y la tecnología. El cuestionario fue diseñado, dirigido y aplicado por investigadores de las universidades ya mencionadas a directores, gerentes y/o propietarios de las empresas. La escala Likert facilitó la valoración de cada uno de los temas. El tema de rendimiento de la empresa se basa en el modelo de Quinn y Rohrbaugh (1983), los

Soto Maciel, A., de la Garza Ramos, M.I., Esparza Aguilar J.L. y San Martín Reyna, J.M. (2015). Evolución de factores competitivos de la empresa familiar ante cambios en la propiedad: el caso mexicano estudiado en Estado de México, Tamaulipas, Quintana Roo y Puebla. Revista de Empresa Familiar, 5 (1), 53-64. 
cuales establecen un modelo espacial de criterios de eficiencia organizacional desde una perspectiva multidimensional y señalan que estos criterios tienen tres dimensiones: la primera, relacionada desde un enfoque interno de la organización (visión sobre el entendimiento y desarrollo de los recursos humanos) a uno externo (nivel macro del éxito de la empresa). La segunda, se basa en la estructura organizativa, haciendo énfasis desde la estabilidad hasta la flexibilidad. La tercera dimensión relacionada con los medios y objetivos organizacionales fue medida a través de una categoría donde el Número 1 equivalía a mínima evolución y el Número 5 a máxima evolución. Los temas de innovación y tecnología fueron asociados a un reactivo $\mathrm{y}$ posteriormente a una evaluación. La categoría que evaluó la innovación se estructuró en cinco niveles donde el 1 correspondía a poco importante y el 5 a muy importante. Mientras que para valorar el uso de la tecnología, el nivel 1 equivalía a poca utilización y el 5 a mucha utilización. Para analizar la evolución de los factores en el tiempo, consideramos tres períodos: los dos últimos años; un periodo de tres a cinco años; y superior a cinco años. Cada uno de estos periodos relaciona respectivamente la probabilidad de un cambio en la propiedad de la empresa: nada probable, probable y muy probable. El análisis estadístico de los datos obtenidos se realizó a través del programa SPSS Versión 21. La validez y fiabilidad de las escalas del cuestionario se verificaron, a través de la validez de contenido, constructo y criterio, así como la consistencia interna de los ítems por medio del coeficiente alfa de Cronbach (1951). El trabajo de campo se realizó durante el periodo comprendido entre diciembre del 2012 y abril del 2013.

\section{Resultados}

Dentro de las características generales de las ochenta y cinco empresas familiares mexicanas que participaron en el estudio se observó que tienen en promedio una antigüedad de 33 años y cuentan con una planta laboral de 72 empleados. La dirección se encuentra predominantemente en la segunda y primera generación (41\% y 39\% respectivamente), la edad promedio de su propietario/gerente es de 54 años y en $88 \%$ de los casos, éste es de género masculino. Las empresas se encuentran registradas principalmente bajo el régimen de persona moral (64\%) y ninguna ha recurrido al modelo de franquicia para su desarrollo.
En cuanto al perfil de su propietario/gerente, los resultados permitieron identificar que en su mayoría han obtenido el grado de licenciatura (46\%) y $15 \%$ de ellos cuentan con un posgrado. Las áreas de Negocios como Contaduría, Administración, Finanzas, Economía, Sistemas Comerciales y Derecho destacan en su preferencia para los estudios de licenciatura y posgrado sobre la Ingeniería (58\% y $28 \%$ respectivamente). En cambio los estudios en Ciencias Sociales y Humanidades o Ciencia y Tecnología tienen un porcentaje menor $(4 \%$ y $6 \%$ respectivamente). El $18 \%$ de los encuestados cuentan únicamente con estudios primarios básicos o de cultura general (22\%), mientras que el $15 \%$ cuenta con bachillerato o alguna formación técnica.

La planta laboral se observa estable tanto para el periodo 2011-2012 como para el 2012-2013. Sin embargo, se identifica una clara tendencia para reducir personal en el segundo periodo, al pasar de $17 \%$ a $35 \%$ de casos. Esta tendencia se confirma con la proyección de crecimiento que pasa del $40 \%$ al $9 \%$ considerando los mismos periodos. La participación laboral de los miembros de la familia se ubica en un promedio de 4 integrantes y se distingue por una clara estabilidad en ambos periodos, seguida por una ligera tendencia a la reducción para el segundo periodo.

Un tema relevante en el estudio es la tendencia sobre el cambio de propiedad de la empresa familiar. Si bien esa eventualidad no es probable dentro de los dos próximos años para $78 \%$ de las empresas, la tendencia es progresiva en el tiempo y esa proporción se reduce de $60 \%$ (dentro de 3 a 5 años) a $45 \%$ (más de 5 años). La proporción de empresas cuyo cambio de propiedad es probable evoluciona de $14 \% 33 \%$ y $23 \%$ respectivamente en los mismos lapsos de tiempo. Una cierta estabilidad se identifica cuando el cambio de propiedad pasa a ser muy probable para los dos primeros periodos (9\%), mientras que se incrementa hasta en $35 \%$ de casos al considerar un periodo superior a 5 años.

Cuando nos interesamos al cambio de propiedad, diferentes escenarios pueden ser posibles. Para $70 \%$ de los casos, la proyección del cambio de la propiedad consiste en que la empresa sea adquirida completamente por la familia; el $12 \%$ proyecta el cambio en la distribución del capital o de las acciones entre los miembros de la familia; el $8 \%$ considera la venta parcial de la empresa; el 5\% su venta total; mientras que solamente en $4 \%$ de los casos se contempla realizar una nueva asociación. En el caso de proyectar la venta parcial de la empresa, los entrevistados se interesan por igual en

Soto Maciel, A., de la Garza Ramos, M.I., Esparza Aguilar J.L. y San Martín Reyna, J.M. (2015). Evolución de factores competitivos de la empresa familiar ante cambios en la propiedad: el caso mexicano estudiado en Estado de México, Tamaulipas, Quintana Roo y Puebla. Revista de Empresa Familiar, 5 (1), 53-64. 
la venta a otra empresa o a un inversionista de capital privado y en menor medida a la venta parcial a un equipo gerencial $(25 \%)$.

\section{Factores competitivos}

Los factores competitivos estudiados fueron: calidad del producto o del servicio; procesos operativos internos; organización de las tareas del personal; satisfacción de los clientes; rapidez de adaptación a las necesidades del mercado; imagen de la empresa y de sus productos o servicios; participación en el mercado; rentabilidad; productividad; satisfacción de los trabajadores; permanencia del personal (reducción de la rotación laboral); y, asistencia y puntualidad del personal (reducción del ausentismo laboral).

Los resultados muestran que la evolución de los factores competitivos en los dos últimos años obtiene un promedio general de 3.9. Los factores que superan este indicador son: calidad del producto o del servicio (4.2); satisfacción de los clientes (4.2); y rentabilidad (4). Contrariamente, entre los factores más marginados aparecen: la permanencia del personal (3.6); la asistencia y puntualidad del personal; y la participación en el mercado (3.7).

Enseguida, se compararon los promedios de los factores precedentes con los promedios de las empresas que proyectan crecimiento en su planta laboral. Los resultados obtenidos arrojan coincidencias en los factores de mayor rezago: permanencia del personal (3.6); y asistencia y puntualidad (3.7). Sin embargo, la evolución de dos factores aparece significativa y positiva: la calidad del producto o del servicio; y la satisfacción de los clientes (4.2).

Otros análisis se realizaron para comparar los niveles de evolución de los factores competitivos en los dos últimos años con tres diferentes escenarios de cambio en la propiedad de la empresa (nada probable, probable y muy probable) y su evolución para tres periodos estudiados (menor de 2 años, de 3 a 5 años y más de 5 años).

Para un periodo inferior de 2 años y cuando el cambio de propiedad es muy probable, los factores competitivos más marginados son: la organización de las tareas del personal, los procesos operativos y la participación en el mercado (3.7). El factor mejor desarrollado es la calidad del producto o del servicio (4.3). Cuando el cambio de propiedad es probable, los factores más marginados que aparecen son: la organización de las tareas del personal y los procesos operativos (3). Mientras que el factor más desarrollado es la satisfacción de los clientes (4.3). Al proyectar estabilidad en la propiedad de la empresa (nada probable el cambio), los factores más marginados son: permanencia del personal (3.3) y organización de las tareas del personal (3.7). Mientras que el factor más evolucionado es la satisfacción de los clientes (4.7).

Comparando los promedios generales de los factores competitivos para los diferentes escenarios de propiedad en este periodo, se observa uniformidad. El mejor desempeño se identifica considerando un probable cambio en la propiedad (4.05), seguido por la conservación de la propiedad (4.02) y el menor rendimiento considerando un muy probable el cambio en la propiedad (4.01).

Para un periodo comprendido entre 3 y 5 años y cuando el cambio de propiedad es muy probable se distinguen la productividad (2.6) y los procesos operativos internos (3.3) como los factores más rezagados. En el sentido opuesto, la calidad del producto o servicio como el factor más desarrollado (4.2). Un cambio probable en la propiedad muestra marginalidad en la asistencia y puntualidad del personal (3.5), así como en la satisfacción de los trabajadores (3.7). Mientras que la calidad del producto o servicio se distingue por su desarrollo (4.3).

En el caso de proyectar estabilidad en la propiedad de la empresa, se observa que los índices de la permanencia del personal (3.7), así como la asistencia y puntualidad del personal (3.8) son los más bajos. El mejor indicador es la calidad del producto o servicio (4.2).

Comparando los promedio generales de los factores competitivos para los diferentes escenarios de propiedad en este periodo, observamos un mejor desempeño cuando se proyecta estabilidad en la propiedad de la empresa (4), mientras que la probabilidad de cambio obtiene 3.8 de promedio $y$ un muy probable cambio en la propiedad se acompaña de 3.5 .

Finalmente, considerando un muy probable cambio en la propiedad para un periodo comprendido superior a los 5 años observamos que los factores competitivos menos desarrollados son la permanencia del personal (3.1), la satisfacción de los trabajadores (3.5) y los procesos operativos internos (3.7), mientras que el más desarrollado corresponden a la calidad del producto o del servicio (4.2).

Cuando el cambio es probable aparecen la productividad (3.6), la permanencia del personal y

Soto Maciel, A., de la Garza Ramos, M.I., Esparza Aguilar J.L. y San Martín Reyna, J.M. (2015). Evolución de factores competitivos de la empresa familiar ante cambios en la propiedad: el caso mexicano estudiado en Estado de México, Tamaulipas, Quintana Roo y Puebla. Revista de Empresa Familiar, 5 (1), 53-64. 
Tabla 1. Correlaciones - Factores competitivos

\begin{tabular}{lcccccc}
\multicolumn{1}{c}{ Factores competitivos } & \multicolumn{2}{c}{ Dentro de 2 años } & \multicolumn{2}{c}{ De 3 a 5 años } & Más de 5 años \\
\cline { 2 - 7 } & $\begin{array}{c}\text { Correlación } \\
\text { de Pearson }\end{array}$ & $\begin{array}{c}\text { Sig. } \\
\text { (bilateral) }\end{array}$ & $\begin{array}{c}\text { Correlación } \\
\text { de Pearson }\end{array}$ & $\begin{array}{c}\text { Sig. } \\
\text { (bilateral) }\end{array}$ & $\begin{array}{c}\text { Correlación } \\
\text { de Pearson }\end{array}$ & $\begin{array}{c}\text { Sig. } \\
\text { (bilateral) }\end{array}$ \\
\hline $\begin{array}{l}\text { Calidad del producto o del } \\
\text { servicio }\end{array}$ & .011 & .924 & .027 & .807 & -.009 & .933 \\
\hline De los procesos operativos & -.037 & .738 & -.198 & .071 & -.212 & .056 \\
\hline $\begin{array}{l}\text { Organización de las tareas del } \\
\text { personal }\end{array}$ & -.053 & .630 & -.074 & .501 & -.155 & .161 \\
\hline Satisfacción de los clientes & -.017 & .876 & $-.232^{*}$ & 0.032 & -.191 & .084 \\
\hline $\begin{array}{l}\text { Rapidez de adaptación a las } \\
\text { necesidades del mercado }\end{array}$ & .088 & .425 & -.190 & .082 & -.145 & .192 \\
\hline $\begin{array}{l}\text { Imagen de la empresa y de sus } \\
\text { productos o servicios }\end{array}$ & .061 & .580 & -.207 & .057 & $-.228^{*}$ & .038 \\
\hline Participación en el mercado & .015 & .889 & -.087 & .426 & -.169 & .127 \\
\hline Rentabilidad & .072 & .517 & -.114 & .302 & -.202 & .069 \\
\hline Productividad & -.016 & .883 & $-.334^{* *}$ & .002 & $-.265^{*}$ & .016 \\
\hline Satisfacción de los trabajadores & .023 & .837 & -.156 & .154 & $-.273^{*}$ & .012 \\
\hline Permanencia del personal & .065 & .554 & -.070 & .525 & $-.258^{*}$ & .018 \\
\hline $\begin{array}{l}\text { Asistencia y puntualidad del } \\
\text { personal }\end{array}$ & .050 & .652 & -.021 & .852 & $-.278^{*}$ & .011 \\
\hline
\end{tabular}

*. La correlación es significativa al nivel 0.05 (bilateral)

**. La correlación es significativa al nivel 0.01 (bilateral)

Fuente: elaboración propia con base en resultados.

los procesos operativos internos (3.7) como los factores menos desarrollados.

Mientras que en el sentido opuesto se observa la calidad del producto o servicio (4.2) y la satisfacción de los clientes (4). En la situación de mayor estabilidad, es decir cuando la empresa familiar está proyectada para ser conservada por el grupo familiar se distingue una gran uniformidad en los factores competitivos. Siendo la satisfacción del cliente (4.3) el factor con el mayor valor y los más débiles, la organización de las tareas del personal (4) y la rapidez de adaptación a las necesidades (3.9).

Comparando los promedios generales de los factores competitivos para los diferentes escenarios de propiedad en este periodo, obtenemos una tendencia decreciente observando que la mayor estabilidad en la propiedad se distingue con un promedio de desempeño de 4.1 , la probabilidad de cambio con 3.8 y la alta probabilidad de cambio con 3.7.

A pesar de que el estudio se interesó en valorar el desempeño a través de doce factores competitivos considerando diferentes proyecciones de propiedad y periodos de tiempo, los resultados muestran tendencias consistentes que llaman la atención. En primer lugar, dos factores competitivos destacan por su frecuencia y representan las principales cualidades de las empresas familiares: la calidad el producto o servicio; y, la satisfacción de los clientes. Estos resultados confirman trabajos precedentes realizados para identificar características distintivas entre empresas familiares y no familiares. En segundo lugar, dos temas se distinguen por representar la mayor debilidad de las empresas estudiadas, los recursos humanos y la organización interna. Dentro de los recursos humanos, los factores más relevantes son: la permanencia del personal; y, la asistencia y puntualidad del personal. Dentro de la organización interna hacemos referencia específicamente a los procesos operativos y a la organización de las tareas del personal.

Otra observación relevante aparece al poner en perspectiva la evolución general de los factores competitivos y la proyección de cambio en la propiedad de la empresa familiar en los distintos periodos, ya que los promedios muestran que el escenario donde la empresa obtiene un mejor desempeño es cuando se proyecta su conservación en la propiedad familiar. De manera opuesta, cuando es muy probable el cambio de la propiedad en la empresa los niveles de desempeño se distinguen por ser los más bajos. Esta situación merece una atención particular en estudios posteriores, pues si bien hay una relación entre un bajo desempeño y la intención de cambio en la propiedad de la empresa, en $70 \%$ de los casos de esta muestra, la expectativa es que pase a manos de la familia.

El Análisis de Correlación de Pearson realizado con los factores competitivos y el cambio de propiedad durante los periodos mencionados, ofrece los siguientes resultados. Una vez calculado el valor del coeficiente de correlación de Pearson interesa

Soto Maciel, A., de la Garza Ramos, M.I., Esparza Aguilar J.L. y San Martín Reyna, J.M. (2015). Evolución de factores competitivos de la empresa familiar ante cambios en la propiedad: el caso mexicano estudiado en Estado de México, Tamaulipas, Quintana Roo y Puebla. Revista de Empresa Familiar, 5 (1), 53-64. 


\begin{tabular}{|c|c|c|c|c|c|c|}
\hline \multirow[b]{2}{*}{ Innovación } & \multicolumn{2}{|c|}{ Dentro de 2 años } & \multicolumn{2}{|c|}{ De 3 a 5 años } & \multicolumn{2}{|c|}{ Más de 5 años } \\
\hline & $\begin{array}{l}\text { Correlación } \\
\text { de Pearson }\end{array}$ & $\begin{array}{c}\text { Sig. } \\
\text { (bilateral) } \\
\end{array}$ & $\begin{array}{l}\text { Correlación } \\
\text { de Pearson }\end{array}$ & $\begin{array}{c}\text { Sig. } \\
\text { (bilateral) } \\
\end{array}$ & $\begin{array}{l}\text { Correlación } \\
\text { de Pearson }\end{array}$ & $\begin{array}{c}\text { Sig. } \\
\text { (bilateral) } \\
\end{array}$ \\
\hline $\begin{array}{lll}\text { En productos o servicios } \\
\text { existentes }\end{array}$ & .226 & .054 & -.138 & .243 & -.253 & .034 \\
\hline $\begin{array}{l}\text { En la comercialización de } \\
\text { nuevos productos o servicios }\end{array}$ & .156 & .230 & $-.288 *$ & 0.24 & $-.341 * *$ & .008 \\
\hline En la gestión empresarial & .250 & .069 & -.240 & .081 & $-.373 * *$ & .006 \\
\hline En los procesos de producción & .233 & 0.79 & -.200 & .132 & $-.411 * *$ & .002 \\
\hline $\begin{array}{l}\text { En las tecnologías de } \\
\text { información y comunicación }\end{array}$ & .048 & .718 & -.205 & .115 & $-.355 * *$ & .006 \\
\hline En la gestión de la calidad & .140 & .254 & -.099 & .423 & $-.417 * *$ & .000 \\
\hline En las compras & -.053 & .689 & -.138 & 298 & -.225 & 0.86 \\
\hline En las ventas & .026 & .845 & -.104 & .431 & $-.320 *$ & .013 \\
\hline
\end{tabular}

determinar si este valor obtenido muestra que las variables estudiadas se encuentran relacionadas o no. En la tabla 1 se observa que los factores competitivos y su relación con la probabilidad de cambio de propiedad, dentro de los próximos 2 años, no arrojaron datos significativos.

Sin embargo, cuando el cambio de propiedad de la empresa se proyecta en un plazo de 3 a 5 años, se observa, tanto en la satisfacción de los clientes como en la productividad, una relación negativa, esto es, en la medida que aumenta una variable disminuye la otra.

En cuanto al cambio de propiedad en más de 5 años, se muestra que la imagen de la empresa y de sus productos y servicios, la productividad, la satisfacción de los trabajadores, la permanencia del personal, y la asistencia y puntualidad, arrojaron datos estadísticamente significativos, lo que indica que estos factores competitivos son afectados por la probabilidad de cambio de propiedad a un mayor plazo.

\section{Innovación}

El estudio de la innovación como factor competitivo primero trató de identificar el área donde ha sido efectiva en los 2 últimos años y posteriormente, se le asoció un nivel de importancia. Las áreas de estudio fueron: en productos o servicios existentes; en la comercialización de nuevos productos o servicios; en la gestión empresarial; en los procesos de producción; en las tecnologías de información y comunicación; en la gestión de la calidad; en las compras; $y$, en las ventas.
Las empresas participando al estudio muestran propensión a la innovación, ya que el $70 \%$ de ellas reconocen haber hecho mejoras. Los resultados muestran que las ocho áreas de estudio han tenido mejoras importantes recientemente y de manera semejante. Las áreas que han experimentado mayores mejoras son las ventas $(79 \%)$ y la gestión de la calidad $(76 \%)$, mientras que las de mayor rezago son la gestión empresarial (63\%) y los procesos de producción (65\%). Estos dos últimos factores coinciden con los resultados precedentes que mostraron la debilidad que representa la organización interna.

En relación a la importancia de la innovación, ésta se sitúa en un promedio de 3.99. Los resultados permiten ordenar decrecientemente la importancia de la innovación en las distintas áreas de la empresa: mejoras en las compras (4.24); en las ventas (4.18); en la gestión de la calidad (4.06); en productos o servicios existentes (4.05); en la comercialización de nuevos productos o servicios (4); en las tecnologías de la información y comunicación (3.85); en los procesos de producción (3.79); y en la gestión empresarial (3.76). Puede observarse que las principales áreas que han sido mejoradas en las empresas se relacionan directamente con las características que aparecieron como distintivas de estas empresas (la calidad del producto o servicio y la satisfacción de los clientes). Mientras que las áreas menos importantes o más marginadas en términos de mejora se relacionan una vez más con la organización interna.

El Análisis de Correlación de Pearson realizado con los factores de innovación y el cambio de 
propiedad durante los periodos mencionados, ofrece los siguientes resultados..

En la tabla 2, se observa que los factores de innovación y su relación con la probabilidad de cambio de propiedad dentro de los próximos 2 años, no arrojaron datos significativos. Sin embargo, en el cambio de propiedad de la empresa dentro de los 3 a 5 años, específicamente en la comercialización de nuevos productos y servicios, los datos son estadísticamente significativos e indica que este factor es afectado por la probabilidad de cambio de propiedad. En relación al cambio de propiedad en más de 5 años, se muestra que en la comercialización de nuevos productos y servicios, en la gestión empresarial, en los procesos de producción, en las TIC, en la gestión de la calidad y en las ventas, arrojaron datos estadísticamente significativos, lo que representa que estos factores son afectados por la probabilidad de cambio de propiedad a un mayor plazo.

\section{Tecnología}

El estudio de las tecnologías de información y comunicación (TIC) como factor competitivo primero trató de identificar las herramientas que poseen las empresas y posteriormente, se les asoció un nivel de utilización en sus operaciones.

Los resultados obtenidos muestran que el 59.8\% de las empresas poseen alguna tecnología de la información y comunicación. Una mayor proporción de empresas disponen de correo electrónico $(76 \%)$, cuentan con una conexión de Internet $(75 \%)$ y mantienen relaciones con sus proveedores y clientes a través de Internet (70\%). Mientras que solo $40 \%$ de ellas utilizan las redes sociales dentro de su gestión y el $49 \%$ realiza mercadotecnia por Internet. Los otros indicadores quedaron ordenados decrecientemente de la siguiente manera: proporción de empresas que disponen de página WEB (56\%) y, realizan compras y ventas por Internet (53\%).

En promedio, el grado de utilización de las tecnologías de la información y comunicación para las empresas estudiadas es de 3.74. El orden decreciente de su utilización coincide con los resultados obtenidos anteriormente sobre su posesión: uso de correo electrónico (4.39); de conexión de Internet (4.27); mantienen relaciones con sus proveedores y clientes a través de Internet (3.98); página WEB (3.79); realizan compras y ventas por Internet (3.47); realizan mercadotecnia por Internet (3.38); y utilizan las redes sociales dentro de su gestión (3.03).

El Análisis de Correlación de Pearson realizado con los factores tecnológicos y el cambio de propiedad durante los periodos mencionados, ofrece los siguientes resultados .

En la tabla 3, se observa que los factores tecnológicos y su relación con la probabilidad de cambio de propiedad dentro de los próximos 2 años, no arrojaron datos significativos.

Sin embargo, en el cambio de propiedad de la empresa dentro de los 3 a 5 años y más de 5, arrojó datos estadísticamente significativos en la empresa

Tabla 3. Correlaciones - Tecnología

\begin{tabular}{|c|c|c|c|c|c|c|}
\hline \multirow[b]{2}{*}{ Tecnología } & \multicolumn{2}{|c|}{ Dentro de 2 años } & \multicolumn{2}{|c|}{ De 3 a 5 años } & \multicolumn{2}{|c|}{ Más de 5 años } \\
\hline & $\begin{array}{l}\text { Correlación } \\
\text { de Pearson }\end{array}$ & $\begin{array}{c}\text { Sig. } \\
\text { (bilateral) }\end{array}$ & $\begin{array}{l}\text { Correlación } \\
\text { de Pearson }\end{array}$ & $\begin{array}{c}\text { Sig. } \\
\text { (bilateral) }\end{array}$ & $\begin{array}{l}\text { Correlación } \\
\text { de Pearson }\end{array}$ & $\begin{array}{c}\text { Sig. } \\
\text { (bilateral) }\end{array}$ \\
\hline $\begin{array}{l}\text { ¿Tiene su empresa conexión } \\
\text { con Internet? }\end{array}$ & $.233^{*}$ & 0.46 & $-.258^{*}$ & .026 & $-.330 * *$ & .005 \\
\hline $\begin{array}{lll}\begin{array}{l}\text { ¿Dispone } \\
\text { electrónico? }\end{array} & \text { de } & \text { correo } \\
\end{array}$ & .148 & .200 & -.055 & .635 & -.174 & .136 \\
\hline ¿Dispone de página WEB? & .202 & .172 & -.179 & .228 & -.152 & .313 \\
\hline $\begin{array}{l}\text { ¿Realiza mercadotecnia } \\
\text { través de Internet? }\end{array}$ & .283 & .077 & -.139 & .393 & -.014 & .934 \\
\hline $\begin{array}{l}\text { ¿Mantiene relaciones con sus } \\
\text { proveedores y clientes a través } \\
\text { de Internet? }\end{array}$ & .125 & .327 & -.180 & .158 & -.203 & .116 \\
\hline $\begin{array}{l}\text { ¿Realiza compras y ventas por } \\
\text { Internet? }\end{array}$ & .139 & .363 & -.061 & .689 & .069 & .657 \\
\hline $\begin{array}{l}\text { ¿Utiliza redes sociales dentro } \\
\text { de su gestión? }\end{array}$ & .200 & .235 & -.224 & .184 & -.238 & .163 \\
\hline
\end{tabular}

Soto Maciel, A., de la Garza Ramos, M.I., Esparza Aguilar J.L. y San Martín Reyna, J.M. (2015). Evolución de factores competitivos de la empresa familiar ante cambios en la propiedad: el caso mexicano estudiado en Estado de México, Tamaulipas, Quintana Roo y Puebla. Revista de Empresa Familiar, 5 (1), 53-64. 
que tiene conexión a internet, lo que indica que estos factores tecnológicos son afectados por la probabilidad de cambio de propiedad a un mayor plazo.

\section{Discusión}

Las empresas familiares mexicanas sufren de gran vulnerabilidad que puede conducirlas a la mortalidad, sobre todo aquellas que se ubican dentro del sector de Pequeñas y Medianas Empresas (PyME). Recientemente, la investigación en gestión se ha interesado en poner en relación a este tipo de empresas, su problemática, su importancia y lo complejo que se vuelve ella cuando se agrega en la ecuación la variable familia. Las empresas de este tipo se enfrentan actualmente a un mercado cada vez más competitivo, más global, mucho más tecnológico en el que tienen que aprender a sobrevivir, a mantenerse en el mercado, independientemente de si son o no familiares.

Los resultados de la empresa familiar en términos de competitividad muestra que su principal dificultad sigue siendo el recurso humano, la parte más valiosa con la que cuentan las empresas el día de hoy, ya que todo lo que puedan desarrollar o generar es parte de la empresa, pero un empleado capacitado, eficiente y con el know how de la empresa solamente es prestado, y si no somos capaces de retener a ese talento humano y continuamos con la rotación de personal que tenemos actualmente, jamás podremos aspirar a mayores niveles de competitividad que nos permitan pasar de pequeña a mediana o de medianas a grandes empresas.

Es importante destacar que las empresas familiares apelan o explotan una de las pocas ventajas competitivas que tienen por ser pequeñas o medianas empresas: su reputación y se cercanía con el cliente. De acuerdo a los resultados, las empresas familiares se preocupan por la calidad de sus productos y/o servicios, por lo que la reputación de la empresa y, por ende, de la familia es muy importante para ellos, están dispuestos a incurrir en costos más altos siempre y cuando el producto entregado tenga la mayor calidad posible, y al preguntar qué es en lo que seguirán invirtiendo en los próximos años, la gran mayoría destaca la calidad ofrecida a sus clientes. Precisamente de aquí se deriva esa segunda ventaja de la que hablábamos, la cercanía con el cliente, este tipo de empresas por lo regular conocen a su cliente, sabe cuáles son sus gustos y preferencias, sabe generar una empatía con ellos, por lo que han logrado contar con su fidelidad generalmente.

Sin embargo, en México la ausencia de una planeación estratégica puede explicar la falta de previsión hacia el futuro y por ende ha impedido capitalizar algunas de las ventajas competitivas de las empresas familiares. En este sentido, es interesante ver cómo los niveles de competitividad cambian conforme se acerca un cambio en la propiedad de la empresa, ya que entre más alejado el cambio en la propiedad de la compañía más irregular el comportamiento en los factores competitivos, es decir, seguimos con una visión de corto plazo, nos preocupamos por el funcionamiento de la empresa en el día a día, pero pareciera que las actividades cotidianas de ésta no nos permiten detenernos a pensar, a realizar una planeación estratégica de largo plazo con la que podamos pasar de ser una empresa reactiva a una empresa preventiva, que encare de mejor manera los problemas y que disminuya el margen de error en la toma de decisiones derivado precisamente de esa mejor planeación.

Precisamente esta estrategia permitirá definir de qué manera la empresa orientará su desarrollo y crecimiento, una vez que se han planteado una visión y objetivos empresariales para el mediano y largo plazo (Ward, 1988). Tal y como Durán y San Martín (2012) lo establecen, sin duda que muchos propietarios, a su vez directores generales de las empresas familiares, tienen en mente la estrategia de su negocio, pero la mantienen de una manera muy personal, asemejándose a lo que Mintzberg (1994) denomina como una estrategia emergente. No obstante la importancia de esta mentalidad estratégica, hace necesario contar con lo que el mismo autor llama una estrategia deliberada, que no restrinja la evolución de la empresa a las capacidades del emprendedor y de su ciclo de vida. La importancia de llevar a cabo esta planeación reside en que, por una parte, la creciente complejidad del negocio exige una mayor participación del equipo directivo, no solamente en la implementación de la estrategia, sino en su formulación. Por otra parte, crear una estrategia deliberada implica verbalizar la estrategia de tal forma que permita alinear las decisiones y acciones del personal de la empresa (Durán y San Martín, 2012).

Es importante destacar lo importante que se torna el que las políticas gubernamentales empiecen a considerar en su diseño el componente familiar de la mayoría de estas empresas. En países como España, Alemania, Chile, entre otros, se ha cobrado

Soto Maciel, A., de la Garza Ramos, M.I., Esparza Aguilar J.L. y San Martín Reyna, J.M. (2015). Evolución de factores competitivos de la empresa familiar ante cambios en la propiedad: el caso mexicano estudiado en Estado de México, Tamaulipas, Quintana Roo y Puebla. Revista de Empresa Familiar, 5 (1), 53-64. 
conciencia de que no es suficiente dirigir políticas de apoyo a las pequeñas y medianas empresas, sino incorporar aspectos que reflejen la incidencia que la familia tiene en su crecimiento y desarrollo. El modelo de capital social de familiness de Pearson et al. (2008) puede ser un buen marco de referencia para ello. Este marco identifica de manera comprensiva los recursos y capacidades que son propios de las empresas familiares, clasificados como estructurales (red de vínculos y organización apropiable), cognitivos (visión y lenguaje compartidos) y relacionales (confianza, normas, obligaciones e identificación).

\section{Conclusiones}

La importancia económica y social de la empresa familiar, así como su alto nivel de vulnerabilidad orientan la necesidad de analizar ciertos factores internos relacionados con la competitividad. El presente trabajo de investigación tuvo como finalidad identificar la existencia, importancia y nivel de evolución que guardan algunos de ellos en las empresas familiares mexicanas considerando diferentes perspectivas temporales.

Las empresas familiares estudiadas se encuentran constituidas como persona moral (64\%), cuentan con 72 empleados (promedio), tienen 33 años de existencia (promedio) y se encuentran bajo la dirección de la segunda generación familiar (41\%). El director general es un varón $(88 \%)$, tiene 54 años de edad (promedio) y cursó estudios de licenciatura $(46 \%)$ en el área de Negocios (58\%). En el periodo 2012-2013 se observa una marcada tendencia negativa, tanto por la proyección de reducción del personal, como por la contracción en su crecimiento. La participación de los miembros de la familia dentro de la empresa se distingue por su estabilidad, al situarse en cuatro personas. El cambio en la propiedad de la empresa que muestran los resultados confirma el deseo expreso de continuidad de la misma, ya que si bien se proyecta un cambio en la propiedad de la empresa a cinco años (55\%), el principal objetivo es convertirla completamente en empresa familiar $(70 \%)$.

Según la Teoría de los Recursos y las Capacidades, la ventaja competitiva es el resultado de una combinación de recursos (heterogéneos, difícilmente imitables y transferibles, valorables y raros) movilizando procesos organizacionales y el saber-hacer colectivo (Barney, 1991; Amit y Schoemaker, 1993). Tomando en cuenta el promedio de evolución de los factores competitivos analizados podemos constatar que la mejora en la calidad del producto o del servicio y la satisfacción de los clientes son los factores principalmente evolucionados en los últimos dos años.

Una observación importante tomando en cuenta las empresas que proyectan crecimiento en su planta laboral, es el interés en mejorar la imagen de la empresa y de sus productos o servicios, así como de la calidad. En cambio, dos factores sobresalen negativamente y de manera recurrente, estos se relacionan con los recursos humanos, concretamente se trata de la rotación del personal y del ausentismo laboral. En menor medida aunque en tendencia también negativa se encuentra la satisfacción de los empleados. Si bien estos factores han experimentado también una evolución en el tiempo, llama la atención que ocupen los últimos lugares dado su relación con la competitividad estratégica por su contribución al saber-hacer colectivo y su difícil reproducción (Polanyi, 1967; Huck y McEwen, 1991; Warren y Hutchinson, 2000).

El desarrollo de los factores competitivos se distingue por ser avanzado y homogéneo cuando la probabilidad de cambio en la propiedad se proyecta dentro de 2 años, de igual manera cuando este no es probable para los dos otros periodos. El mismo fenómeno se observa de manera inversamente proporcional. El desarrollo de los factores competitivos es contraído e irregular cuando no se proyecta cambio dentro de 2 años y cuando éste es muy probable para los dos otros periodos. Una posición competitiva fundada en los recursos es el resultado de la unidad de un conjunto de activos estratégicos que la empresa posee y valora en productos o mercados (Dierickx y Cool, 1988). En las empresas de este estudio, dos de las áreas que más han sido mejoradas, las compras y las ventas, no constituyen una fuente de ventaja competitiva duradera. En cambio, las áreas más rezagadas, la gestión empresarial y los procesos de producción se encuentran en el centro de los procesos organizacionales que son los responsables de combinar los recursos de la empresa para convertirlos en ventaja competitiva duradera. Esta situación revela en las empresas una debilidad importante, probablemente relacionada con una visión estratégica limitada y de corto plazo.

Para la teoría movilizada, la eficiencia actual de la empresa depende de su elección estratégica que es circunscrita por su propia historia y de sus fenómenos sociales que son complejos (Itami, 1987). Las TIC muestran una existencia y un grado de utilización heterogéneo en las empresas. Si bien

Soto Maciel, A., de la Garza Ramos, M.I., Esparza Aguilar J.L. y San Martín Reyna, J.M. (2015). Evolución de factores competitivos de la empresa familiar ante cambios en la propiedad: el caso mexicano estudiado en Estado de México, Tamaulipas, Quintana Roo y Puebla. Revista de Empresa Familiar, 5 (1), 53-64. 
de manera general se observa un amplio uso en sus operaciones, todavía falta mucho por hacer. Potencialmente, un grado de utilización mayor del correo electrónico, la conservación de relaciones con proveedores y clientes por Internet y realizar mercadotecnia por internet cuyo resultado es inferior al promedio pueden asociarse a una mayor contribución en el desarrollo de ventajas competitivas duraderas. En fin, se reconoce ampliamente que las empresas más competitivas son aquellas que tienen una gestión estratégica capaz de vehicular recursos, competencias, capacidades organizacionales y activos estratégicos que se maximizan económicamente en el tiempo. Esta lógica debería de guiar la toma de decisiones de las empresas familiares mexicanas.

Nuevas líneas de investigación aparecen pertinentes e interesantes para ser exploradas, como la relación económica de los factores competitivos ante cambios en la propiedad de la empresa familiar. Así como el conocimiento de las lógicas y los procesos implicados en la evolución de los factores competitivos, probablemente a través de la Perspectiva relacional de la Teoría de los Recursos y las Capacidades (Dyer y Singh 1998).

\section{Referencias}

Alvarez, J. y García, E. (1996). Factores de éxito y riesgo en la pyme: diseño e implantación de un modelo para la mejora de la competitividad, Economía Industrial, $310,149-161$.

Amit R. y Schoemaker P. (1993). "Strategic assets and organizational rent", Strategic Management Journal, 14, $33-46$.

Arrègle J. (1995). Le savoir et l'approche Resource Based: une ressource et une compétence", Revue Française de Gestion, Octobre.

Barney J. (1991). "Firm resources and sustained competitive advantage", Journal of Management, 17(1), 90-102.

Black J. y Boal K. (1994). Strategic resources: traits, configurations and paths to sustainable competitive advantage. Strategic Management Journal, 15, 131-148.

Cabrera, K. y García, J. (1998). Cambios en la cultura y estructura de las empresas frente a las tecnologías de la información empírica. VIII Congreso Nacional sobre Empres Familiar de la Asociación Científica de Economía y Dirección de la Empresa (ACEDE), Universidad de las Palmas de la Gran Canaria, España.

Camelo, C., Lorenzo D., Martin F. y Valle R. (1999). Competitividad regional y recursos intangibles: un análisis comparado Andalucía-España, Servicio de
Publicaciones de la Universidad de Cádiz.

Cronbach, L. (1951). Coefficient alpha and the internal structure of test. Psychometrika, 22(3), 297-334.

Dierickx, I. y Cool, K. (1989). Asset Stock Accumulation and Sustainability of Competitive Advantage, Management Science, 35(12), 1504-1511.

Dyer J. y Singh H. (1998). "The relational view: cooperative strategy and sources of interorganizational competitive advantage", Academy of Management Review, 23(4), 660-679.

Donovan, W. (1996). Can technology really help small business? Small Business Forum, 14(2), 77-78.

Durán J., San Martín J., Montiel H., (2012). A research proposal to examine entrepreneurship in family business, Journal of Entrepreneurship, Management and Innovation, 8(3), 58-77.

Esparza, J. L. y Duréndez, A. (2011). "Los sistemas de control de gestión y su efecto en el rendimiento de las empresas familiares mexicanas: un estudio empírico para el sector turístico", Revista de Economía No. 76 (UADY), México. http://www.revista.economia.uady.mx/

Gallo, M. (1995). La empresa familiar. Texto y casos. Editorial Praxis, S. A. Barcelona.

Grant R. (1996). "Toward a knowledge-based theory of the firm", Strategic Management Journal, 17, 109-122.

Hamel G. y Prahalad C. (1990). "The core competence of the corporation", Harvard Business Review, 68(3), 7991.

Hernández, R. Fernández, C. y Baptista, P. (2006). Metodología de la investigación, McGraw-Hill, México.

Itami H. (1987). Mobilizing invisible assets, Harvard Business Press.

Lansberg, I. y Astrachan, J. (1994). Influence of family relationships on succession planning and training. The importance of mediating factors. Family Business Review, 7(1), 39-59.

Luck, S. (1996). Success in hong Kong: factores selfreported by successful small business owners, Journal of Small Business Management, 34(3), 68-75.

Métais E. (2004). Stratégie et ressources de l'entreprise, Economica, Paris.

Mintzberg H. (1994). The rise and fall of strategic planning, New York: Free Press.

Neubauer, F., y Lank, A. (1999). La empresa familiar. Cómo dirigirla para que perdure. Editorial Deusto. Bilbao.

North, D., Smallbone, D. y Vickers, I. (2001). Public sector support for innovating SME's, Small Business Economics, 16, 303-317.

Pearson A., Carr, J. and Shaw, J. C. (2008). Toward a theory of families: a social capital perspective.

Soto Maciel, A., de la Garza Ramos, M.I., Esparza Aguilar J.L. y San Martín Reyna, J.M. (2015). Evolución de factores competitivos de la empresa familiar ante cambios en la propiedad: el caso mexicano estudiado en Estado de México, Tamaulipas, Quintana Roo y Puebla. Revista de Empresa Familiar, 5 (1), 53-64. 
Entrepreneurship theory and practice, X(X), 949-969.

Penrose E. (1959). The theory of the growth of the firm. John Wiley. New York.

Polanyi, K. (1967). The tacit dimension, Garden City, New York.

Quinn, R.E. y Rohrbaugh, J. (1983). “A spatial model of effectiveness criteria: towards a competing values approach to organizational analysis". Management Science, 29(3), 363-377.

Soto A. (2007). La constitution et les effets du capital social des dirigeants dans les réseaux interorganisationnels: le cas du système productif local de Méca Bourg, Tesis Doctoral, Université Jean Moulin Lyon 3.

Stalk G., Evans P. y Shulman E. (1992). "Competing on capabilities: the new rules of corporate strategy", Harvard Business Review, 70(2), 54-65.

Teece D., Pisano G. y Shuen A. (1997). "Dynamic capabilities and strategic management", Strategic Management Journal, 8(7), 509-533.
Van Auken, H. Madrid A. y García Perez de Lema, D. (2008). Innovation and performance in Spanish manufacturing SME's, International Journal of Entrepreneurship and Innovation Management, 8(1), 3656.

Vermeulen, P. (2004). Managing product innovation in financial service firms, European Management Journal, 22(1), 43-50.

Viedma, J. (1990). La excelencia empresarial española, Viama Editores, Barcelona.

Ward, J. (1988). The special role of strateging planning for family businesses. Family Business Review, 1(2), 105-117.

Wernerfelt B. (1984). "A Resource-based View of the Firm”, Strategic Management Journal, 5(2), 171-180.

Williamson O. (1996). The mechanisms of governance, Oxford, University Press, Oxford.

Wortman M.S. Jr. (1994). Theoretical foundations for family-owned business: a conceptual and research -based paradigm, Family Business Review, Journal of the Family Firm Institute, 7(1), 3-27. 